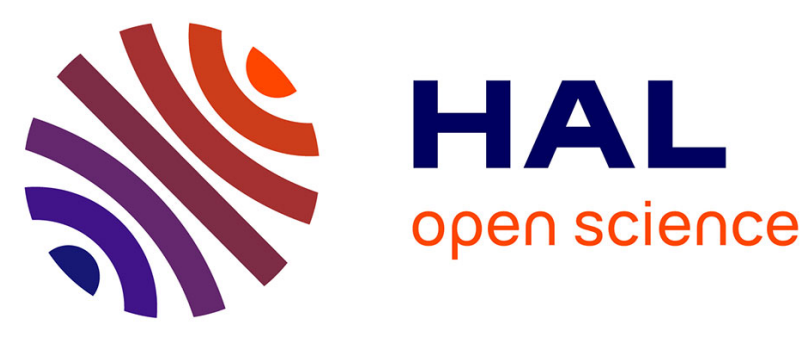

\title{
Room-temperature spin nutations in a magnetically condensed phase of $[\mathrm{Y}(\mathrm{pc}) 2] \bullet$
}

Athanassios K Boudalis, Jorge Enrique Olivares Peña, Eufemio Moreno

Pineda, Artem Fediai, Wolfgang Wenzel, Philippe Turek, Mario Ruben, Jorge-Enrique Olivares-Peña, Eufemio Moreno-Pineda

\section{To cite this version:}

Athanassios K Boudalis, Jorge Enrique Olivares Peña, Eufemio Moreno Pineda, Artem Fediai, Wolfgang Wenzel, et al.. Room-temperature spin nutations in a magnetically condensed phase of $[\mathrm{Y}(\mathrm{pc}) 2] \bullet$. Chemical Communications, 2021, 10.1039/D1CC05491K . hal-03375127

\section{HAL Id: hal-03375127 \\ https://hal.science/hal-03375127}

Submitted on 12 Oct 2021

HAL is a multi-disciplinary open access archive for the deposit and dissemination of scientific research documents, whether they are published or not. The documents may come from teaching and research institutions in France or abroad, or from public or private research centers.
L'archive ouverte pluridisciplinaire HAL, est destinée au dépôt et à la diffusion de documents scientifiques de niveau recherche, publiés ou non, émanant des établissements d'enseignement et de recherche français ou étrangers, des laboratoires publics ou privés. 


\section{Room-temperature spin nutations in a magnetically condensed phase of $\left[\mathrm{Y}(\mathrm{pc})_{2}\right]^{\circ}$}

Received 00th January 20xx, Accepted 00th January 20xx DOI: $10.1039 / \times 0 \times x 00000 x$

\author{
Athanassios K. Boudalis, ${ }^{* a, b, c}$ Jorge-Enrique Olivares-Peña, ${ }^{a}$ Eufemio Moreno-Pineda, ${ }^{d}$ Artem \\ Fediai, ${ }^{a}$ Wolfgang Wenzel, ${ }^{a}$ Philippe Turek, ${ }^{e}$ Mario Ruben*a, ${ }^{a, c}$
}

FID-detected nutations of the antiferromagnetic crystal form of $\left[\mathrm{Y}(\mathrm{pc})_{2}\right]^{\bullet}$ demonstrated that its radical spin can be coherently driven in its magnetically condensed undeuterated phase and at room temperature. Liquid-helium nutations revealed additional Rabi oscillations assigned to transitions within higher-multiplicity states of finite-sized chain fragments.

Organic radicals have emerged as viable candidates for Quantum Information Processing (QIP) applications, in particular as potential spin qubits. Indeed, their low spin-orbit couplings endow them with weak interactions with their lattice, leading to slow spin-lattice relaxation (large $T_{1}$ times), which may favour longer decoherence times $\left(T_{2}\right)$, a favourable characteristic for spin qubits.

Moreover, organic radicals offer us the arsenal of organic chemistry to rationally design single- and multiqubit architectures. As molecular objects, they are excellent candidates to solve the limitation in precisely positioning other qubit types based on defect qubits (e.g., nitrogen vacancies in diamonds) or impurities (e.g., ${ }^{31} \mathrm{P}$ in ${ }^{28} \mathrm{Si}$ ).

However, a challenge of organic radicals is their chemical stability; indeed, many organic radicals are air-sensitive, which not only complicates their spectroscopic characterization but also makes them less than ideal candidates for inclusion in devices. Moreover, surface deposition in high-purity films, such as achieved by ultra-high vacuum (UHV) thermal evaporation is not always a technique of choice, as not all organic radicals exhibit the requisite thermal stability. Finally,

\footnotetext{
a. Karlsruhe Institute of Technology (KIT), Institute of Nanotechnology, EggensteinLeopoldshafen, Germany.E-mail: mario.ruben@kit.edu

${ }^{b}$ Institute for Quantum Materials and Technology (IQMT), Karlsruhe Institute of Technology (KIT), Eggenstein-Leopoldshafen, Germany.

. Institut de Science et d'Ingénierie Suparamolaiculaires - ISIS, 8 allée Gaspard Monge, BP 70028, F-67083 Strasbourg Cedex, France. E-mail:

bountalis@unistra.fr

${ }^{d .}$ Departamento de Química-Física, Escuela de Química, Facultad de Ciencias

Naturales, Exactas y Tecnología, Universidad de Panamá, Panamá.

e. Institut de Chimie de Strasbourg (UMR 7177, CNRS-Unistra), Université de

Strasbourg, 4 rue Blaise Pascal, CS 90032, F-67081 Strasbourg, France.

Electronic Supplementary Information (ESI) available: [details of any supplementary information available should be included here]. See DOI: $10.1039 / x 0 x x 00000 x$
}

several radical types are accessible through complicated synthetic processes, which is an additional drawback.

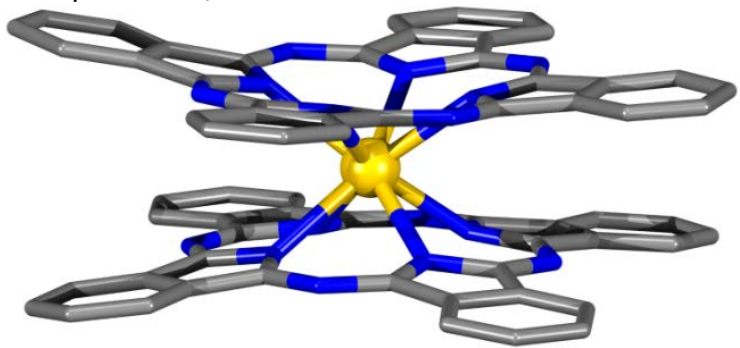

Figure 1. Crystal structure of $\left[\mathrm{Y}(\mathrm{pc})_{2}\right]^{\circ}$. Colour code: $\mathrm{C}$, grey; $\mathrm{N}$, blue; $\mathrm{Y}$, gold.

Among radical compounds, graphenoid biradicals have demonstrated coherence times of $290 \mu \mathrm{s}$ in frozen $\mathrm{CS}_{2}$ solutions $(80 \mathrm{~K}),{ }^{1}$ whereas a CNOT gate experiment has been developed and experimentally tested on a photogenerated biradical in a frozen solution $(85 \mathrm{~K})$. $^{2}$ Previously, a TEMPO biradical $^{3}$ and a Blatter-type radical ${ }^{4}$ were studied along these lines, yielding room-temperature nutation signals in the solid state. However, both were magnetically diluted by embedding in a diamagnetic matrix: a non-radical host and mesoporous silica matrix, respectively. Expanding on other spin-qubit types, room-temperature Rabi oscillations in the solid state had also

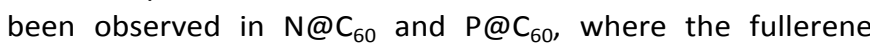
constitutes a diamagnetic host, ${ }^{5}$ in $\mathrm{Cr}^{5+}$ diluted in $\mathrm{K}_{3} \mathrm{NbO}_{8}{ }^{6}$ and the metal complex $\left[\mathrm{V}^{\mathrm{IV}} \mathrm{O}(\mathrm{pc})\right]$ diluted in diamagnetic [TiO(pc)] at $1: 10 .^{7}$ To our knowledge, no coherent nutations have been reported in magnetically condensed phases of spin qubits at room temperature. These precautions are usually accompanied by isotopic engineering, such as ligand deuteration, to decrease the amount of magnetic nuclei, which are also known to accelerate decoherence.

With these in mind, we set out to exploit our experience in the domain of the coordination chemistry of aromatic macrocycles, such as phthalocyanines and porphyrins, which are known to give access to highly stable and sublimable metal complexes. As an example, the neutral complex $\left[\mathrm{Tb}(\mathrm{pc})_{2}\right]$, comporting a radical $\mathrm{pc}^{\cdot-}$ ligand, has been shown to be extremely amenable to a wide variety of surface deposition techniques for the creation of numerous spintronic devices. ${ }^{8}$ 



Figure 2. FID-detected EPR spectra of $\left[\mathrm{Y}(\mathrm{pc})_{2}\right]^{\circ}$ at $295 \mathrm{~K}$ (top) compared to the CW spectrum plotted in absorption mode (bottom). The $g$-scale is plotted from the $H$-scale derived by considering a $2.80 \mathrm{MHz} / \mathrm{G}$ correspondence. Exp. Conditions. $\mathrm{CW}$ spectrum $f_{\mathrm{MW}}=9.32186 \mathrm{GHz}, \Delta B_{\text {mod }}=0.1 \mathrm{Gpp}, P_{\mathrm{MW}}=4.69 \mu \mathrm{W}$. FID spectrum: $f_{\mathrm{MW}}=9.73914 \mathrm{GHz}$.

To harness the potential of the radical spin system, we focused on complex $\left[\mathrm{Y}(\mathrm{pc})_{2}\right]^{\cdot}$, carrying a diamagnetic $\mathrm{Y}^{\prime \prime \prime}$ ion and an $S=$ $1 / 2$ radical spin delocalized over the two ligands. In the solid state, this complex is known to crystallise in two forms: the solvated $\left[\mathrm{Y}(\mathrm{pc})_{2}\right]^{\circ} \cdot \mathrm{CH}_{2} \mathrm{Cl}_{2}$ form, known to crystallise in the Pnma space group, giving rise to ferromagnetic linear chains, and the desolvated $P 2_{1} 2_{1} 2_{1}$ form forming antiferromagnetic such chains. ${ }^{9,10}$ While it was reported ${ }^{9,10}$ that the desolvated $P 22_{1} 2_{1} 2_{1}$ form is isostructural to its $\mathrm{Nd}^{\prime \prime \prime}$ analogue, a full structural characterization for the $\mathrm{Y}^{\prime \prime \prime}$ analogue was only reported in 2009 (CCDC code VUKZAD). ${ }^{11}$

The AF nature of the desolvated form was further corroborated by variable-temperature solid-state ${ }^{1} \mathrm{H}-\mathrm{NMR} .{ }^{12}$ Apart from a CW HFEPR spectrum on the Pnma solvated form, ${ }^{13}$ no other EPR studies have been carried out on either forms of $\left[\mathrm{Y}(\mathrm{pc})_{2}\right]^{\circ}$. For a preliminary assessment of its solidstate EPR behaviour of we carried out CW and pulsed EPR studies at the X-band on an undeuterated sample.

Powder XRD data agreed to those predicted for the desolvated $P 2{ }_{1} 2_{1} 2_{1}$ structure, confirming the sample's phase purity. The solid-state sample at $295 \mathrm{~K}$ yielded a simple isotropic signal in $\mathrm{CW}$ mode, similar to that reported for the solvated phase. ${ }^{13}$ In pulsed mode it demonstrated an FID and no echos, typical of a homogeneously broadened species. CW spectra plotted in absorption mode appear broader than the FT=spectra of the on-resonance FIDs (Figure 1, assuming a correspondence of $2.80 \mathrm{MHz} / \mathrm{G}$ for the resonance field and frequency of the CW spectrum). We attribute this to the dead time after the $\pi / 2$ pulse which hinders the observation of fast-decaying FIDs, corresponding to broader lines. Attempts to record field-swept FID-integral-detected spectra were complicated by the rectangular pulse shapes, giving rise to sinc sidelobes which deformed the spectra (see $\mathrm{SI}$ ). Interestingly, cooling causes a line broadening (see $\mathrm{SI}$ ), from $0.43 \mathrm{mT}$ at $295 \mathrm{~K}$ to $0.53 \mathrm{mT}$ at 5 K (FWHM). This aligns with the relatively faster decay of the FID at $5 \mathrm{~K}$ (see below and SI). However, exchange-related phenomena might also influence the overall lineshapes. In any case, these line widths are in agreement with previously reported HFEPR of the FM form. ${ }^{13}$

Monoexponential fits to the FID at $295 \mathrm{~K}$ yielded $T_{\mathrm{m}}=0.11 \mu \mathrm{s}$, whereas FID-detected inversion recovery experiments, fitted with a stretched exponential function, yielded a spin-lattice relaxation time of $T_{1}=0.135 \mu \mathrm{s}(B=0.87$, see Supporting Information).

We also undertook FID-detected spin nutations to determine whether the spins of this radical could be coherently driven by microwave pulses. Surprisingly, very clear Rabi oscillations were recorded (Figure 3). As revealed by fits to a single exponentially damped oscillation, their frequency varied linearly to the intensity of $\left|\mathbf{B}_{1}\right|$, i.e., to $\sqrt{ } P_{\mathrm{MW}}$. These fits are shown as 3D lines superimposed onto the FT spectra (Figure 4 ), and separately in the SI. The FT spectra reveal a single peak for each attenuation.

We then tested the behavior of $\left[\mathrm{Y}(\mathrm{pc})_{2}\right]^{\bullet}$ at $5 \mathrm{~K}$. Although the spin lattice relaxation time increases slightly $\left(T_{1}=0.192(6) \mu \mathrm{s}\right.$, $B=0.84)$, the phase-memory time actually shows a decrease $\left(T_{\mathrm{m}}=0.087 \mu \mathrm{s}\right)$.

Nutation experiments at $5 \mathrm{~K}$ also yielded Rabi oscillations (Figure 5) which, however, could not be fitted to a single frequency. At each MW power, apart from the main frequency $f_{1}(P)$, FT spectra revealed clear but weaker peaks at frequencies $f_{2}(P) \approx 2 f_{1}(P)$, and even weaker peaks at frequencies $f_{3}(P) \approx 3 f_{1}(P)$ (Figure 6).

This qualitative assessment was further corroborated by fits of the nutation traces to multiple exponentially damped oscillations. Fits to a single oscillation were of particularly poor quality. They were remarkably improved by inclusion of a second frequency and further still improved by the inclusion of a third one. Inclusion of a fourth frequency still afforded an improvement, albeit marginal (see SI for $10 \mathrm{~dB}$ fit). Like in the case of the $295 \mathrm{~K}$ data, the best-fit values of the derived Rabi frequencies were also linear to $\left|\mathbf{B}_{1}\right|$ (Figure 6 and $\mathrm{SI}$ ).

As to the physical meaning of those additional peaks, we recall that the Rabi frequency for a resonance between states $M_{S}$ and $M_{S}+1$ within a multiplet $S$, is given by: ${ }^{14}$

$f_{\text {nut }}=\frac{\mu_{B} g B_{1}}{\hbar} \sqrt{S(S+1)-M_{S}\left(M_{S}+1\right)}$

Given this dependence, we can assign the basic frequency $f_{1}$, to the $M_{S}= \pm 1 / 2$ transition of an $S=1 / 2$ doublet, whereas integer multiples are assigned to states of higher multiplicities. Thus, frequency $2 f_{1}$ arises from the $M_{S}= \pm 1 / 2$ transition within an $S=3 / 2$ multiplet, frequency $3 f_{1}$ within an $S=5 / 2$ multiplet, etc. In fact, when plotted on a logarithmic ordinate, the $5 \mathrm{~K} \mathrm{FT}$ spectra revealed a series of weaker peaks with multiple frequencies $n f_{1}(P)$. Due to overlaps, a precise assignment was not possible, but clear peaks appeared at frequencies corresponding to high $n$ values, e.g., $n=15-17$ for the $10 \mathrm{~dB}$ spectrum (Figure 7).

We attribute such higher-multiplicity states to antiferromagnetically exchange-coupled fragments of $\left[\mathrm{Y}(\mathrm{pc})_{2}\right]^{\bullet}$ radicals which are formed in the condensed state. Indeed, it has been shown that this complex forms chains in the solid state. Crystal defects would break the chains to fragments of finite sizes containing $m$ radicals each. 


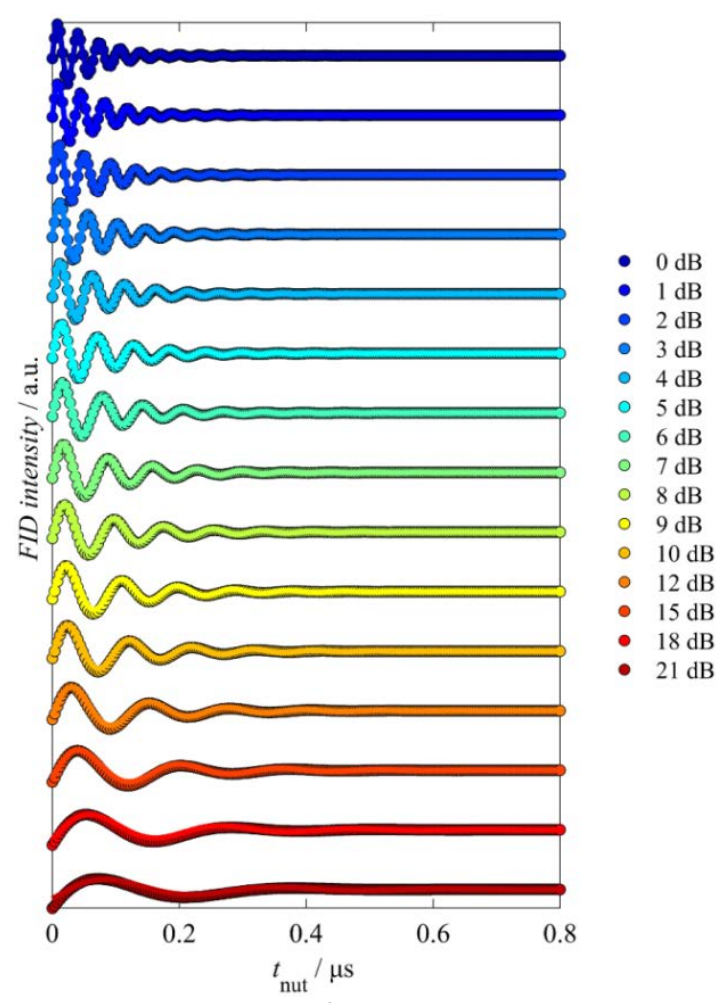

Figure 3. Rabi oscillations of $\left[\mathrm{Y}(\mathrm{pc})_{2}\right]^{\circ}$ at $295 \mathrm{~K}$. The lines are fits to a single exponentially damped oscillation.

These would be characterized by a spin ladder of multiplets reaching up to $S_{\mathrm{T}}=m / 2$. Odd-membered fragments would constitute Kramers systems yielding $M_{S}= \pm 1 / 2$ resonances at perpendicular-mode EPR, with nutations of frequencies $n f_{1}$, where $n=(m+1) / 2$. Thus, a $n=15$ frequency multiple would correspond to a transition within a $S_{\mathrm{T}}=29 / 2$ multiplet. Since a frequency multiple $n$ will only be observed for fragments of

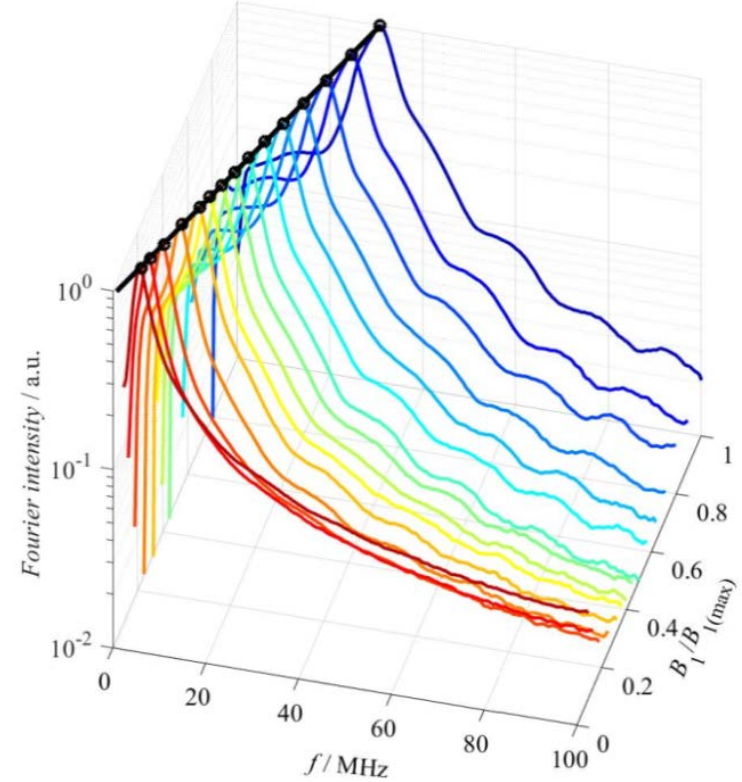

Figure 4. FT spectra of nutation traces at $295 \mathrm{~K}$. The superimposed scatter plot represents the Rabi frequencies best-fit values to the original data, considering a single exponentially damped oscillation. The line is a linear fit to these four sets of frequencies. Error bars correspond to $\pm 1 \sigma$ confidence intervals, and are, for the most part, too small to be visible.

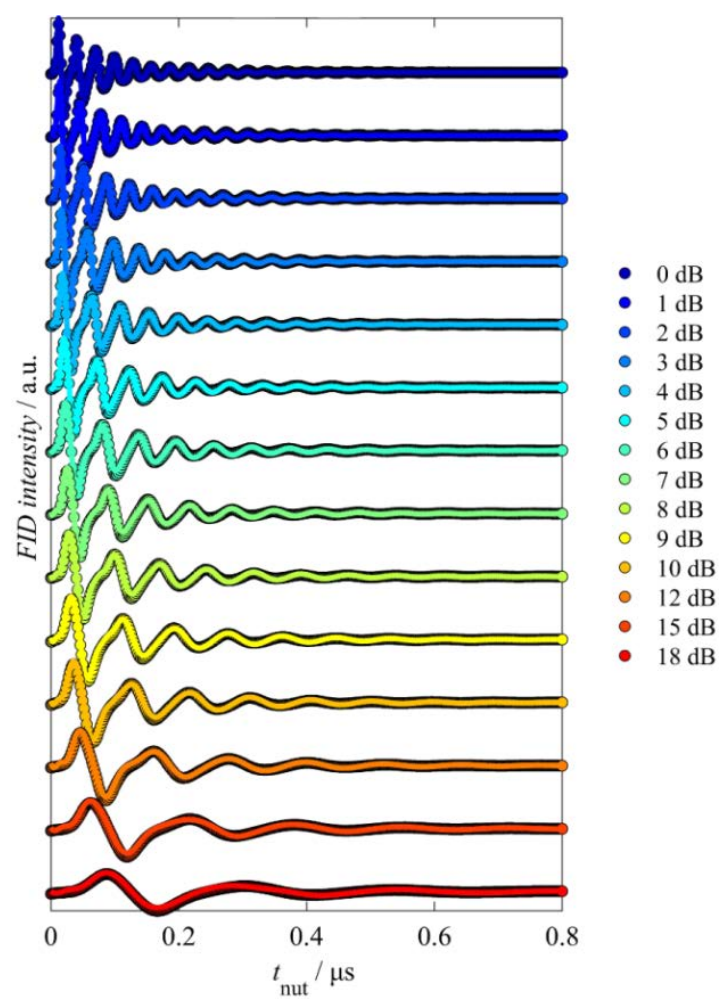

Figure 5. Rabi oscillations of $\left[\mathrm{Y}(\mathrm{pc})_{2}\right]^{\bullet}$ at $5 \mathrm{~K}$. The lines are fits to four exponentially damped oscillations of frequencies $f_{1}, 2 f_{1}, 3 f_{1}$ and $4 f_{1}$.

nuclearities of $2 n-1$ and above, such peaks are weaker, as crystal defects make larger fragments progressively less probable. In light of the fact that the sample is $A F$, the appearance of these additional Rabi oscillations only at the $5 \mathrm{~K}$ experiments, seems counter-intuitive. Indeed, if this was due to thermal depopulation upon cooling, the higher harmonics due to the excited high-multiplicity resonances should actually

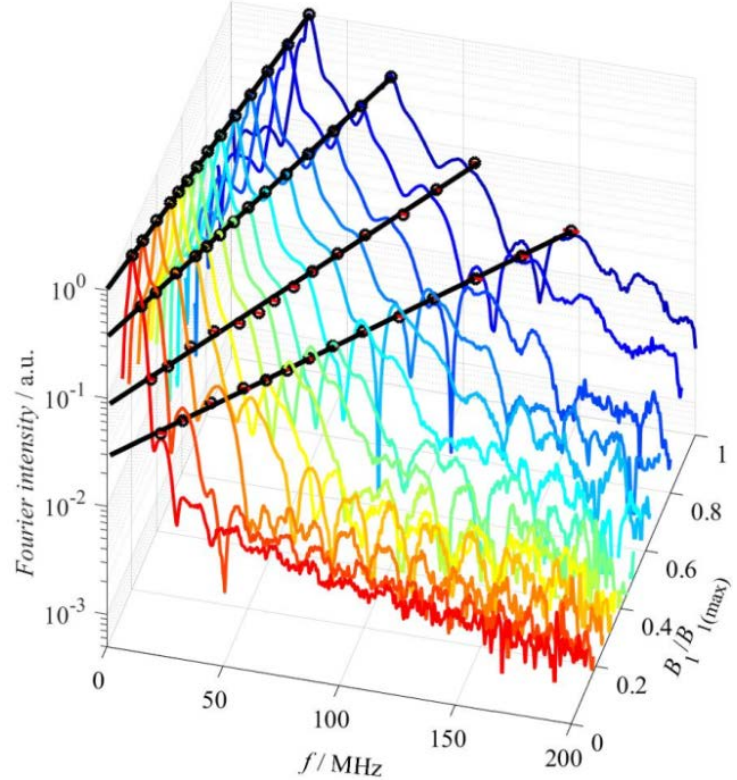

Figure 6. FT spectra of nutation traces at $5 \mathrm{~K}$. The superimposed scatter plots represent the Rabi frequencies best-fit values to the original data, considering four exponentially damped oscillations. The lines are linear fits to these four sets of frequencies. Error bars correspond to $\pm 1 \sigma$ confidence intervals, and are, for the most part, too small to be visible. 


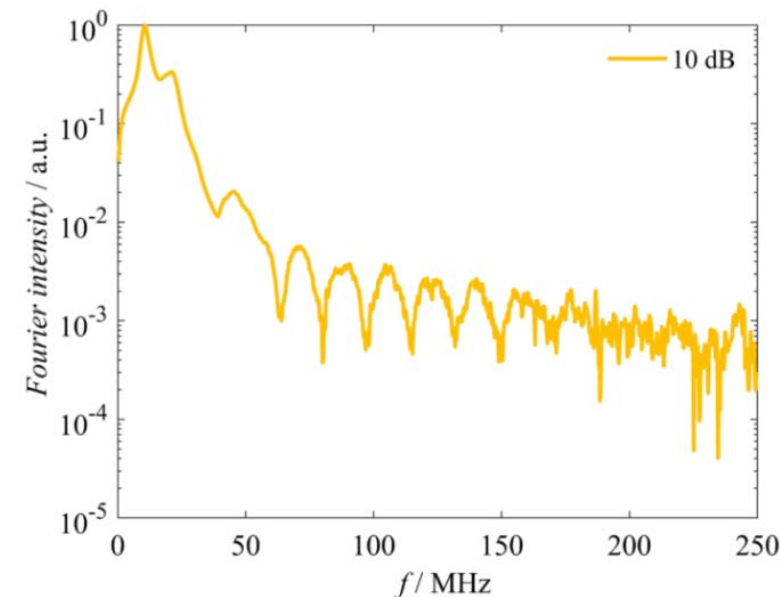

Figure 7. FT spectrum of the $10 \mathrm{~dB}$ trace, plotted on a logarithmic ordinate.

decrease in intensity.

This means that this behaviour is not due to a thermal depopulation of excited states, since it is precisely those excited $S>1 / 2$ states that would become thermally depopulated upon cooling. Besides, given the strength of the magnetic coupling $\left(J=-4.78 \mathrm{~K}\right.$ assuming $\mathrm{a}-J \hat{\mathbf{S}}_{i} \hat{\mathbf{s}}_{j}$ Hamiltonian formalism) a multitude of states would remain equally populated even at $5 \mathrm{~K}$; e.g. for the $m=5$ fragment, Boltzmann population analysis of the $\left|S, M_{S}\right\rangle$ states suggests a relative population of 0.037 for the ground $|1 / 2,-1 / 2\rangle$ state and 0.030 for the excited $\mid 5 / 2,-1 / 2>$ one at $0.34 T$ of the EPR experiment. Instead, we assign this behaviour to the different temperature profiles of the Rabi time of each transition specifically we suggest that the Rabi times of transitions from higher multiplicities drop off much more rapidly upon heating, practically disappearing at room temperature.

To provide a qualitative rationalization of these experimental results, we performed Density Functional (DFT) calculations on [YPc $\left._{2}\right]^{\circ}$ chains of $m=3$ and 5 radicals (see SI for details). Analysis of the DFT results reveals that the electrons on the spin densities arise due to the carbon atoms on the ligands which can be also observed on the Projected Density of States (SI). Moreover, periodic simulations performed using the crystal structure of the complex crystallizing in the $P 2_{1} 2_{1} 2_{1}$ phase ${ }^{11}$ yielded a DFT ground state indicating FM interactions $\left(m=3: S_{\mathrm{T}}=3 / 2 ; m=5: S_{\mathrm{T}}=5 / 2\right)$. However, calculations on a box with empty spaces, to simulate magnetically isolated chain fragments (Figure 8), led to an AF coupling ( $m=3: S_{\mathrm{T}}=1 / 2 ; m=$ 5: $S_{\mathrm{T}}=1 / 2$ or $3 / 2$ ) with a clear energy separation between spin states. These results, which are in line with experimental observations, indicate that the AF interactions may be intimately related to the magnetic isolation between chains.

In conclusion, we report the first demonstration of Rabi oscillations in a radical system at the least favourable conditions, i.e., in its magnetically condensed phase, in a nondeuterated sample and at room temperature. This observation creates new perspectives for the use of this, or similar, systems in QIP applications in practically appealing conditions. Moreover, the low-temperature study reveals the input from higher-multiplicity spin states characteristic of the extended nature of the sample at hand, also a first in our experience. There are no conflicts of interest to declare.

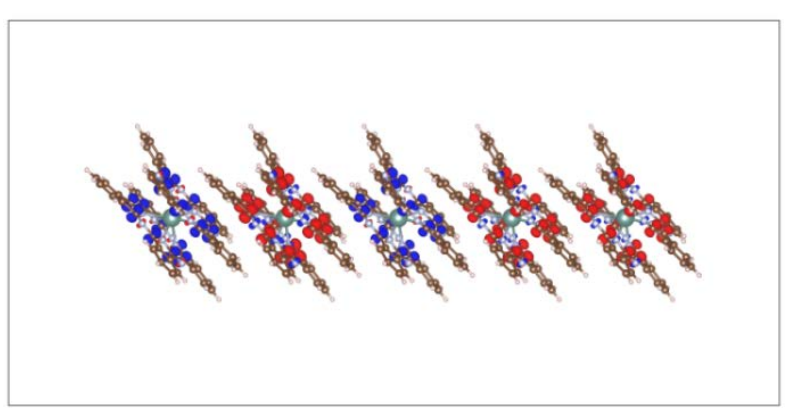

Figure 8. Spin density and box border (black lines around the molecules) of the $m=5$ chain fragment. The $\alpha / \beta$ spins are shown in red/blue, respectively.

The authors wish to thank Nathalie Gruber for acquisition of XRD data. They also acknowledge the Baden-Württemberg Stiftung for financial support through the MOLTRIQUSENS project and the REseau NAtional de Rpe interDisciplinaire (RENARD, Fédération RPE CNRS \#3443) for support with experiments. EMP thanks the Panamanian National Systems of Investigators (SNI, SENACYT) for support.

\section{Notes and references}

1 F. Lombardi, A. Lodi, J. Ma, J. Liu, M. Slota, A. Narita, W. K. Myers, K. Müllen, X. Feng and L. Bogani, Science, 2019, 366, 1107-1110.

2 J. N. Nelson, J. Zhang, J. Zhou, B. K. Rugg, M. D. Krzyaniak and M. R. Wasielewski, J. Chem. Phys., 2020, 152, 014503.

3 S. Nakazawa, S. Nishida, T. Ise, T. Yoshino, N. Mori, R. D. Rahimi, K. Sato, Y. Morita, K. Toyota, D. Shiomi, M. Kitagawa, H. Hara, P. Carl, P. Höfer and T. Takui, Angew. Chem. Int. Ed., 2012, 51, 98609864.

4 A. S. Poryvaev, E. Gjuzi, D. M. Polyukhov, F. Hoffmann, M. Fröba and M. V. Fedin, Angew. Chem. Int. Ed., 2021, 60, 8683-8688.

5 C. Meyer, W. Harneit, B. Naydenov, K. Lips and A. Weidinger, Appl. Magn. Reson., 2004, 27, 123-132.

6 S. Nellutla, K.-Y. Choi, M. Pati, J. van Tol, I. Chiorescu and N. S. Dalal, Phys. Rev. Lett., 2007, 99, 137601.

7 M. Atzori, L. Tesi, E. Morra, M. Chiesa, L. Sorace and R. Sessoli, Journal of the American Chemical Society, 2016, 138, 2154-2157.

8 A. K. Boudalis, K. S. Kumar and M. Ruben, in Reference Module in Chemistry, Molecular Sciences and Chemical Engineering, Elsevier, 2021, p. B9780081026885001000.

9 J. L. Paillaud, M. Drillon, A. De Cian, J. Fischer, R. Weiss and G. Villeneuve, Phys. Rev. Lett., 1991, 67, 244-247.

10 J. L. Paillaud, M. Drillon, A. Decian, J. Fischer, R. Weiss, R. Poinsot and A. Herr, Physica B: Condensed Matter, 1991, 175, 337-348.

11 K. Katoh, Y. Yoshida, M. Yamashita, H. Miyasaka, B. K. Breedlove, T. Kajiwara, S. Takaishi, N. Ishikawa, H. Isshiki, Y. F. Zhang, T. Komeda, M. Yamagishi and J. Takeya, J. Am. Chem. Soc., 2009, 131, 9967-9976.

12 F. Branzoli, P. Carretta, M. Filibian, S. Klyatskaya and M. Ruben, Phys. Rev. B, 2011, 83, 174419.

13 D. Komijani, A. Ghirri, C. Bonizzoni, S. Klyatskaya, E. MorenoPineda, M. Ruben, A. Soncini, M. Affronte and S. Hill, Physical Review Materials, 2018, 2, 024405.

14 A. Schweiger and G. Jeschke, Principles of pulse electron paramagnetic resonance, Oxford University Press, Oxford, UK ; New York, 2001. 